\title{
Survey of HER2-neu Expression in Colonic Adenocarcinoma in the West of Iran
}

\author{
Seyed-Hamid Madani ${ }^{1}$, Edris Sadeghi ${ }^{2 *}$, Akram Rezaee ${ }^{1}$, Masoud Sadeghi ${ }^{3}$, \\ Sedigheh Khazaee ${ }^{1}$, Nasrin Amirifard ${ }^{2}$, Mehrdad Payandeh ${ }^{3}$
}

\begin{abstract}
Background: Overexpression of HER2-neu has been reported in many epithelial malignancies, including cancers of the breast, ovaries, lungs, prostate, bladder, pancreas, colorectum and stomach as well as osteosarcomas. The aim of this study was evaluation of expression of HER2-neu immunohistochemistry (IHC) status and clinicopathologic features in a series of colonic adenocarcinomas. Materials and Methods: In this descriptive and analytical study, we surveyed 211 samples of colon adenocarcinoma from 182 patients $(86.3 \%)$ undergoing total or partial colectomy and $29(\mathbf{7 . 1 3 \%})$ with biopsies by colonoscopy. A sufficient sample size was obtained from all cases and the slides were stained with hematoxylin and eosin and also by IHC (HER2) staining. Results: The mean age for the patients at diagnosis was $\mathbf{5 7 . 9}$ years (range, 15-88 years). One hundred and twenty one patients $(57.3 \%)$ were male. Of all patients, 201 samples $(95.3 \%)$ were conventional adenocarcinomas $(159,29$ and 13 cases were well, moderately and poorly differentiated, respectively) and $10(4.7 \%)$ were mucinous type. Out of 211 cases, 171 were checked for lymph nodes metastasis and 64 were positive. There is a correlation between HER 2 scores and differentiation, most score 3 cases being well differentiated $(\mathbf{P}<0.05)$. Conclusions: In patients with advanced colon cancer, surgery alone is not curative and other forms of therapy may be required to prolong patient survival. HER2 overexpression was found in some cases and this could be a guideline to new adjuvant therapy for these patients.
\end{abstract}

Keywords: Colon cancer - Her2-neu - IHC - differentiation - Iran

Asian Pac J Cancer Prev, 16 (17), 7671-7674

\section{Introduction}

Colorectal cancer is the fourth most common cancer in men and the third most common in women (Payandeh et al., 2015).The HER2-neu proto-oncogene is 1 of 4 epidermal growth factor receptor (EGFR)-related receptors. The HER 2-neu gene is located on chromosome $17 \mathrm{q} 21$ and encodes a $185 \mathrm{kDa}$ transmembrane protein with tyrosine kinase activity that functions as a growth factor receptor (Akiyama et al., 1986). Overexpression of HER2-neu has been reported in many epithelial malignancies, including cancers of the breast (Andersen et al., 1995), ovarian (Kono et al., 2000), lungs, prostate, bladder, pancreas, osteosarcoma, colorectal and gastric adenocarcinomas (Farzand et al., 2014). While the tyrosine kinase family receptors are found on normal cells, there is evidence that they are overexpressed in many types of tumors (Rusch et al., 1993).

Remarkably, some studies reported both membranous and cytoplasmic overexpression with much higher rates up to $60 \%$ (Golijow et al., 2001). The reported prevalence of HER 2-neu overexpression in colon cancer ranges from 0 - 83\% [Ross and McKenna,2001; Caruso and Valentini,1996]. In a group of studies containing a smaller number of cases, the rate of gene amplification has been reported to be in the range of $0-30 \%$ (Golijow et al., 2001). It is reported that monoclonal antibody based kits have a better agreement with HER2 gene amplification, as determined by FISH (fluorescence in situ hybridization). Generally, the 3+ cases have an excellent concordance with FISH, while the $2+$ results are more equivocal. Current guidelines demand a FISH-analysis to determine the gene amplification in 2+ cases (Blok et al., 2013). Several antibodies have been developed for treatment of CRC and were clinically tested. Three mAbs, cetuximab (erbitux, anti-EGF receptor [EGFR], panitumumab (Vectibix ${ }^{\circledR}$, antiVEGF) and bevacizumab (Avastin ${ }^{\circledR}$, ant i-VEGF), have been extensively studied for the treatment of CRC and, indeed, were shown to be effective (Nazemalhosseini et al., 2013). Patterns are scored as immunohistochemical (IHC) 0 (no staining or staining in $<10 \%$ of tumor cells, negative), IHC 1+ (faint/barely perceptible incomplete membrane staining in $>10 \%$ of tumor cells, negative), IHC $2+$ (weak to moderate complete membrane staining

${ }^{1}$ Molecular Pathology Research Center, Imam Reza Hospital, ${ }^{2}$ Cancer Research Center, ${ }^{3}$ Medical Biology Research Center, Kermanshah University of Medical Sciences, Kermanshah, Iran*For correspondence: sadeghi_mkn@yahoo.com 


\section{Seyed-Hamid Madani et al}

in $>10 \%$ of tumor cells, equivocal), or IHC $3+$ (strong complete membrane staining in $>10 \%$ (until 2007) or $>30 \%$ (2007-now) of tumor cells, positive) (Wolff et al., 2007). The aim of this study is evaluation of expression of HER2-neu IHC status and clinicopathologic features in colonic adenocarcinoma.

\section{Materials and Methods}

In this descriptive and analytical study, we survey 211 samples for colon adenocarcinoma analysis included 182 patients $(86.3 \%)$ of the total or partial colectomy and 29 $(7.13 \%)$ biopsies that were obtained by colonoscopy test.

\section{Clinical and pathologic evaluation}

First of all, we provided instruments, apparatus, materials (like Canon Powershot G6) and chemistry compounds (like Hydrogen peroxide, Entelan glue made in Germany (Merk)), kits and colors (c-erbB-2 oncoprotein A*0485, Biotin Blocking System*X0590, EnVision+Dual Link System-HRP*K4063+Liguid DAB+Substrate Chromogen System*K3468, Target Retrieval Solution, pH9 $(\times 10) *$ S2367 made in Denmark and Hematoxylin made in Spain). After that, we prepared stock buffer (mixing ethylenediaminetetraacetic acid (EDTA) (0.4gr) with tris(hydroxymethyl)aminomethane (Tris) (2gr) and distilled water ( D.W) $(1000 \mathrm{ml})$ and for the IHC staining, first we charged slides with poly-L-lysin glue means that the slides were incubated for an hour in the glue 10 percent

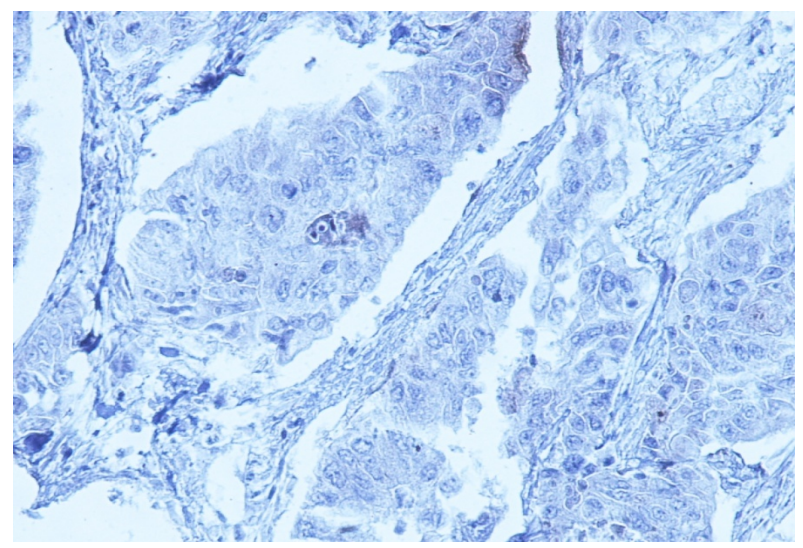

Figure 1. IHC staining (×100) marker HER2-neu (0) in Colonic Adenocarcinoma

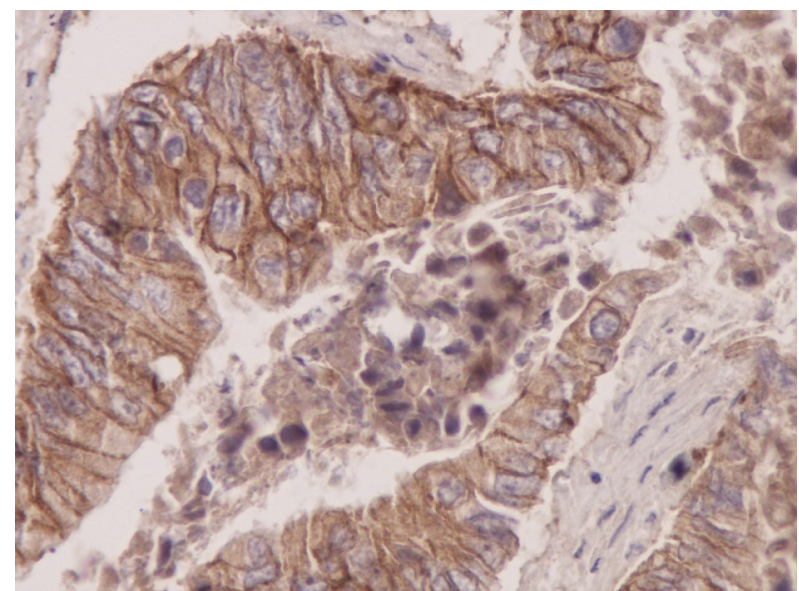

Figure 3. IHC staining (×100) marker HER2-neu (2+) in Colonic Adenocarcinoma
(10 ml, $90 \mathrm{ml}$ of distilled water plus glue) and then dried at room temperature overnight. In this study, the patients admitted to pathology laboratory of Imam Reza and Biston Hospitals in Kermanshah (Iran) that were diagnosed colon adenocarciunoma for them a pathologist, a sufficient sample size was selected from any patient and the slides were stained by hematoxylin and eosin (H \& E) method. Then the samples in paraffin blocks of 4 micron sections were prepared for staining $\mathrm{H} \& \mathrm{E}$ and then re-cut and prepared for IHC (HER2) staining.

\section{Statistical analysis}

Statistical analyses were performed using the SPSS statistical software package version 19 (SPSS, Chicago, IL, USA). Chi-square test was used to analyze the significance of correlation between the expression of HER2 and clinicopathological parameters. P-value $<0.05$ was considered significant.

\section{Results}

The mean age for the patients at diagnosis was 57.9 years (range, 15-88 years). One hundred and twenty one patients $(57.3 \%)$ were male and ninety patients $(42.7 \%)$ were female. Based on histology of patients with adenocarcinoma of the colon, 201 samples (95.3\%) of them were conventional and $10(4.7 \%)$ of them were mucinous adenocarcinoma type. We survey just conventional adenocarcinoma of the colon $(n=201)$ for

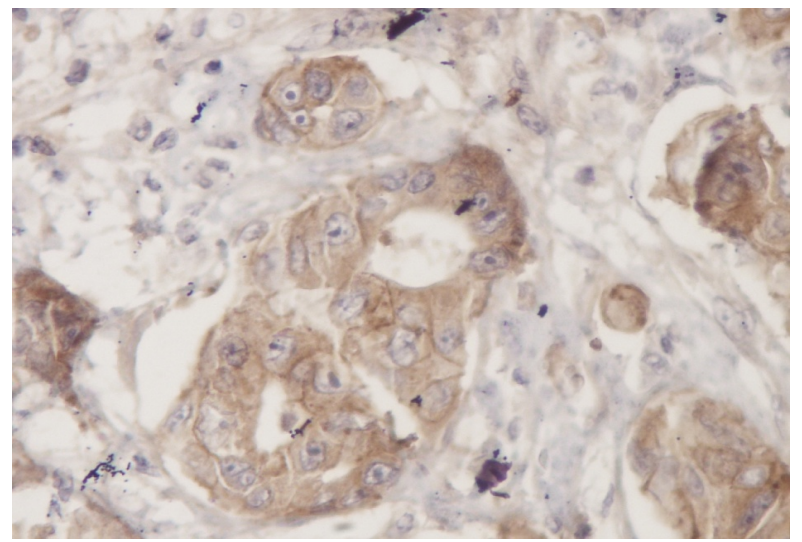

Figure 2. IHC staining (×100) marker HER2-neu (1+) in Colonic Adenocarcinoma

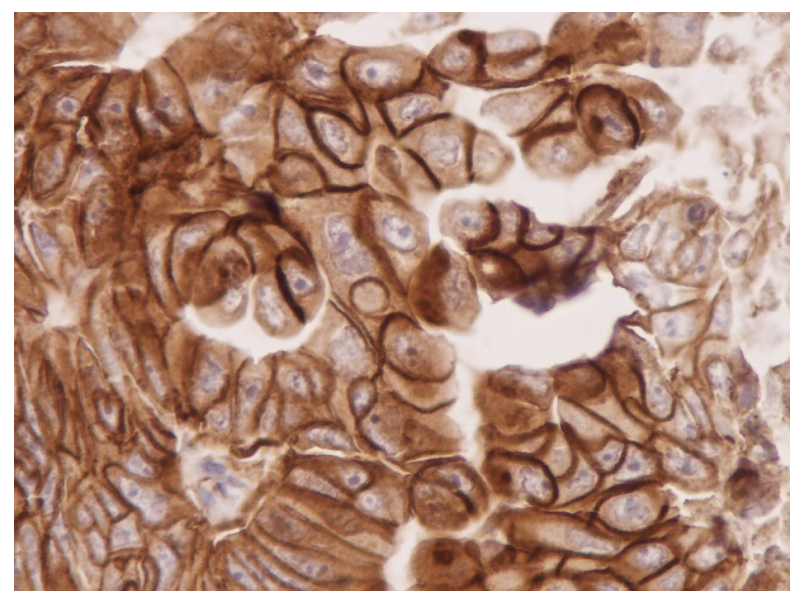

Figure 4. IHC staining (×100) Marker HER2-neu (3+) in Colonic Adenocarcinoma 
Table 1. The Baseline Characteristics for all Patients

\begin{tabular}{lrcc}
\hline Variables & $\mathrm{N}(\%)$ & Mean & Range \\
\hline Age(year) & & 57.9 & $15-88$ \\
Sex & & & \\
$\quad$ Male & $121(57.3)$ & & \\
$\quad$ Female & $90(42.7)$ & & \\
Tumor Subtype & & & \\
$\quad$ Conventional & $201(95.3)$ & & \\
$\quad$ Mucinose & $10(4.7)$ & & \\
Tumor Differentiation & & \\
$\quad$ Well & $159(79.1)$ & \\
$\quad$ Moderately & $29(14.4)$ & \\
$\quad$ Poorly & $13(6.5)$ & \\
HER2-neu Score & & \\
0 & $42(19.9)$ & \\
1 & $68(32.3)$ & \\
2 & $75(35.5)$ & \\
3 & $26(12.3)$ & \\
\hline
\end{tabular}

Table 2. The Correlation between HER2-neu Scores and a Number of Variables

\begin{tabular}{lcccc}
\hline Variables, $\mathrm{n}(\%)$ & Score 0 and 1 & Score 2 & Score3 & P-value* \\
\hline Sex & & & & 0.104 \\
Male & $61(50.4)$ & $49(40.5)$ & $11(9.1)$ & \\
Female & $49(54.4)$ & $26(28.9)$ & $15(16.7)$ & \\
Tumor Subtype & & & & 0.496 \\
$\quad$ Conventional & $103(51.2)$ & $73(36.3)$ & $25(12.4)$ & \\
$\quad$ Mucinose & $7(70)$ & $2(20)$ & $1(10)$ & \\
Tumor Differentiation(n=201) & & & 0.003 \\
$\quad$ Well & $71(44.7)$ & $66(41.5)$ & $22(13.8)$ & \\
$\quad$ Moderately & $20(69)$ & $7(24.1)$ & $2(6.9)$ & \\
$\quad$ Poorly & $12(92.3)$ & $0(0)$ & $1(7.7)$ & \\
Age group & & & & 0.914 \\
$\quad$ Age $\leq 60$ & $62(53.4)$ & $40(34.5)$ & $14(12.1)$ & \\
Age $>60$ & $48(50.5)$ & $35(36.8)$ & $12(12.6)$ & \\
Involved Lymph Node (n=171) & & & 0.097 \\
$\quad$ Yes & $44(68.8)$ & $15(23.4)$ & $5(7.8)$ & \\
$\quad$ No & $56(52.3)$ & $41(38.3)$ & $10(9.3)$ & \\
\hline
\end{tabular}

*Chi-square test

tumor differentiation that $159(79.1 \%), 29(14.4 \%)$ and $13(6.5 \%)$ cases were differentiated well, moderately and poorly, respectively. Out of 211 cases in this report, 171 cases were checked for lymph nodes metastasis that $107(57 \%)$ cases had without metastasis and 64 $(30.3 \%)$ cases had metastasis. IHC staining of HER2 protein is scored qualitatively as $0 / 1+/ 2+/ 3+$ with $42(19.9 \%) / 68(32.2 \%) / 75(35.5 \%) / 26(12.3 \%)$ values in our patients (Table 1). IHC staining of HER-2 protein for score 0 , score $1+$, score $2+$ and score $3+$ has been shown in Figures 1, 2, 3, and 4, respectively.

There was no significant relation between clinicopathologic variables (sex, age of the patients, tumor subtype and involved lymph node) with HER2 overexprission $(\mathrm{P}>0.05)$. There is the correlation between HER2 scores with well differentiated tumor $(\mathrm{P}<0.05)$ (Table 2).

\section{Discussion}

IHC and FISH results in cohort A corresponded well. Almost all primary tumors with an IHC grade of 0 or $1+$ showed no amplification by FISH, whereas all IHC 3+ cases showed amplification. Of the IHC $2+$ cases, only about one-sixth showed HER2 amplification (Kim et al., 2011). Therefore, reliable separation of IHC $1+/ 0$ and IHC 2+ may be difficult in biopsy samples, and FISH analysis should be used for definitive classification (Shan et al., 2013). The prevalence of HER2-neu overexpression in colon cancer detected by IHC has been previously reported between 0 and 83\% (Maurer et al., 1998; Porebska et al., 2000). A study examined 317 colon cancer samples for the presence of HER2-neu oncoprotein by immunohistochemistry. In all, 49 samples $(15.5 \%)$ showed positive expression of HER2-neu; only 7 samples were strongly membrane positive (Li et al., 2011). Similar results were described in other study that authors examined HER2-neu protein expression in 132 CRC specimens, and found HER2-neu overexpression in $11 \%$, with 2 displaying were strong membranous immunostaining (Kavanagh et al., 2009). In another study, strong membranous HER-2 staining was detected in only $5 \%$ of tumors in 96 primary human colorectal adenocarcinomas that also showed HER-2 gene amplification (Half et al., 2004). In a small study (77 specimens) indicating a very low rate of HER-2-/neu positivity (Schuell et al., 2006). We examined 211 colon cancer samples that $26(12.3 \%$ ) (Score 3+) case of them had HER2-neu overexpression. Two studies reported that the HER2-neu expression was not correlated with sex, age, tumor differentiation, localization of the primary tumor and overall survival (Jesus et al., 2005; Schuell et al., 2006) and also was indicated that HER2-neu expression was unlikely to play a major role in the therapeutic management of colorectal cance (Jesus et al., 2005). A study conducted in Iran in 2006 to evaluate association between HER2-neu protein overexpression and tumor grades was also highly significant (Leung et al., 2008). Other study (Farzand et al., 2014) found significant correlation between HER2 positive overexpression (cytoplasmic staining) and tumor differentiation, as is in our study. Also, two studies did not find any correlation between HER2-neu protein overexpression and lymph node metastasis (McKay et al., 2002; Farzand et al., 2014). The HER2-neu overexpression or the gene amplification was also not associated with any specific clinicopathological features (Nathason et al., 2003; Schuell et al., 2006). In our study, we did not find significant relation between clinicopathologic variables (sex and age of the patients, tumor subtype, involved lymph node) with HER2 overexprission ( $\mathrm{P}>0.05)$, but we found correlation between HER2 scores with well differentiated tumor $(\mathrm{P}<0.05)$. We survey adenocarcinoma of the colon $(n=201)$ for tumor differentiation that $79.1 \%, 14.4 \%$ and $6.5 \%$ percent were differentiated well, moderately and poorly, respectively. In a study these percents were $66.7 \%, 36 \%$, respectively and in higher grades, HER2-neu staining decreased (Leung et al., 2008). A research found a significant association between Her2neu protein expression in colorectal adenocarcinomas and lymph node metastases (Sun et al., 1994) while we did not find any significant relationship between these factors.

In conclusion, in these patients with advanced colon cancer, surgery alone is not curative and other forms of therapy may be required to prolong patient survival. HER2 


\section{Seyed-Hamid Madani et al}

overexpression was found in some patients and this could be a guideline to new adjuvant therapy for these patients. .

\section{References}

Akiyama T, Sudo C, Ogawara H, Toyoshima K, Yamamoto $\mathrm{T}$ (1986). The product of the human c-erbB-2 gene: a 185-kilodalton glycoprotein with tyrosine kinase activity. Science, 232, 1644-6.

Andersen TI, Paus E, Nesland JM, McKenzie SJ, Børresen AL (1995). Detection of c-erbB-2 related protein in sera from breast cancer patients. Relationship to ERBB2 geneamplification and c-erbB-2 protein overexpression in tumour. Acta Oncol, 34, 499-504.

Blok EJ, Kuppen PJ, van Leeuwen JE, Sier CF (2013). Cytoplasmic Overexpression of HER2: a Key Factor in Colorectal Cancer. Clin Med Insights Oncol, 7, 41-51.

Caruso ML, Valentini AM (1996). Immunohistochemical p53 overexpression correlated to c-erbB-2 and cathepsin D proteins in colorectal cancer. Anticancer Res, 16, 3813- 8.

Farzand S, Siddique T, Saba K, Bukhari MH (2014). Frequency of HER2/neu overexpression in adenocarcinoma of the gastrointestinal system. World J Gastroenterol, 20, 5889-96.

Golijow C, Guerci A, Mouron S, et al (2001). Activation of $\mathrm{K}$-ras and c-erbB-2 protooncogenes in human colonic adenocarcinomas. Acta Gastroenterol Latinoam, 31, 71- 6.

Half E, Broaddus R, Danenberg KD, et al(2004). HER-2 receptor expression, localization, and activation in colorectal cancercell lines and human tumors. Int J Cancer, 108, 540-8.

Jesus EC, Matos D, Artigiani R, et al (2005). Assessment of staging, prognosis and mortality of colorectal cancer by tumor markers: receptor erbB-2 and cadherins. Acta Cir Bras, 20, 422-27.

Kavanagh DO, Chambers G, O'Grady L, et al (2009). Is overexpression of HER-2 a predictor of prognosis in colorectal cancer? BMC Cancer, 9, 1-6.

Kim MA, Lee HJ, Yang HK, Bang YJ, Kim WH(2011). Heterogeneous amplification of ERBB2 in primary lesions is responsible for the discordant ERBB2status of primary and metastatic lesions in gastric carcinoma. Histopathology, 59, $822-31$.

Leung SP, Griffith OL, Masoudi H, et al (2008). Clinical utility of type 1 growth factor receptor expression in colon cancer. Am J Surg, 195, 604-10.

Li Q, Wang D, Li J, Chen P (2011). Clinicopathological and prognostic significance of HER-2/neu and VEGF expression in coloncarcinomas. BMC Cancer, 27, 11, 277.

Maurer CA, Friess H, Kretschmann B, et al (1998). Increased expression of erbB3 in colorectal cancer is associated with concomitant increase in the level of erbB2. Hum Pathol, 29, 771-7.

McKay JA, Loane JF, Ross VG, et al (2002). c-erbB-2 is not a major factor in the development of colorectal cancer. $\mathrm{Br} \mathrm{J}$ Cancer, 86, 568-73.

Nathason DR, Culliford AT, Shia J, et al (2003). HER-2/neu expression and gene amplification in colon cancer. Int $J$ Cancer, 105, 796-802.

Nazemalhosseini Mojarad E, Kuppen PJ (2013). HER2 and immunotherapy using monoclonal antibodies in colorectal cancer. Immunotherapy, 5, 1267-9.

Payandeh M , Sadeghi M, Sadeghi E, Gholami F (2015). Analysis of KRAS, BRAF and NRAS in Patients with Colorectal Cancer: the First Report of Western Iran. American J Cancer Prev, 3, 9-22.

Porebska I, Harlozinska A, Bojarowski T (2000). Expression of the tyrosine kinase activity growth factor receptors (EGFR, ERB B2, ERB B3) in colorectal adenocarcinomas and adenomas. Tumour Biol, 21, 105-15.

Ross JS, McKenna BJ(2001). The HER-2/neu oncogene in tumors of the gastrointestinal tract. Cancer Invest, 19, 554-68.

Rusch V, Baselga J, Cordon-Carlo, et al (1993). Differential expression of the epidermal growth factor receptor and its ligand in primary non-small cell lung cancers and adjusent benign lung. Cancer Res, 53, 2379-85.

Schuell B, Gruenberger T, Scheithauer W, Zielinski Ch, Wrba F (2006). HER 2/neu protein expression in colorectal cancer. BMC Cancer, $\mathbf{6}, 123$.

Shan L, Ying J Lu (2013). HER2 expression and relevant clinicopathological features in gastric and gastroesophageal junction adenocarcinoma in a Chinese population. Diagn Pathol, 8, 76.

Sun XF, Carstensen JM, Sta 1 O, Zhang H, Nordenskjold B (1996). Proliferating cell nuclear antigen (PCNA) in relation to ras, c-erbB-2,p53, clinico-pathological variables and prognosis in colorectal adenocarcinoma. Int J Cance, 69, 5-8.

Wolff AC, Hammond MEH, Schwartz JN, et al (2007). American society of clinical oncology/college of American pathologists guideline recommendations for human epidermal growth factor receptor 2 testing in breast cancer. Archives of Pathology and Laboratory Medicine, 131, 18-43. 